\title{
Chiasma distribution, genetic lengths, and recombination fractions: a comparison between chromosomes 15 and 16
}

\author{
NINA SAADALLAH AND MAJ HULTÉN \\ From the Regional Cytogenetics Laboratory, East Birmingham Hospital, Bordesley Green East, Birmingham \\ B9 5ST.
}

SUMMARY The chiasma distribution of bivalents 15 and 16 identified at diakinesis by a quadruple staining technique including DA-DAPI fluorescence has been investigated in two human males. The $\Theta$ study has shown that chiasmata are not distributed at random. Both chromosomes have distally localised chiasmata, but in the long arm of chromosome 15 chiasmata are also found to be localised proximally, adjacent to the centromere. Genetic lengths and recombination fractions have been calculated from chiasma distribution data for the major bands of chromosomes 15 and 16 under the assumptions that there is no chromatid interference, no chiasma movement, and no difference between mitotic and meiotic band positions. The localisation of chiasmata implies much discrepancy in recombination patterns between the acrocentric chromosome 15 and the submetacentric chromosome 16.

Investigations on meiotic chiasmata provide a unique cytogenetic opportunity to gain insight into patterns of recombination. Under the assumptions that there is no chiasma movement and no chromatid interference, chiasma distribution data may be used to estimate both the genetic map interval and the recombination fraction between any chromosomal markers, as recently described for chromosomes 1 , 2 , and 9 by Hultén $e t$ al ${ }^{1}$ and Laurie et $a .^{2}{ }^{2}$

A prerequisite for the analysis is obviously that individual chromosomes and chromosome arms can be unequivocally identified, which imposes some practical problems because chromosome identification is more difficult at meiosis than at mitosis. Thus, at diakinesis/first metaphase, the centromeres cannot be reliably visualised by staining techniques such as orcein, Giemsa, or $\mathbf{Q}$ banding because the centromeric primary constriction is absent; therefore C banding is obligatory. Secondly, at this stage, chromosome configurations are more complex than at mitosis with crosses and rings resulting from chiasma formation after pairing between homologues, which in itself complicates the interpretation of the $Q$ banding patterns. It should also be noted that although the band locations at mitosis and meiosis are basically the same, ${ }^{3}$ the meiotic bivalents

Received for publication 17 January 1983. Accepted for publication 19 January 1983. at diakinesis/first metaphase are inherently more condensed, resulting in a reduced resolution of individual bands.

Some chromosomes such as D14 and D15 may not be easy to differentiate unambiguously in any one cell, even after consecutive $\mathrm{Q}$ and $\mathrm{C}$ banding, ${ }^{4}$ and we have therefore applied other staining techniques on human male meiotic chromosomes to improve this situation. As expected from the experience with mitotic cells, the Distamycin-DAPI fluorescence turns out to be the most useful as regards identification of D15 and also specifies E16 with certainty. We have therefore incorporated the DADAPI into a quadruple staining technique with $Q$ and $\mathrm{C}$ banding and cresyl fast violet. The initial $Q$ staining allows differentiation between the long and short arms of chromosome 16 and the subsequent DA-DAPI staining means that chromosomes 15 and 16 may be easily and reliably identified. Cresyl fast violet gives the chromosomes a sharper outline, which is useful for drawing and measuring the chiasma locations, and finally $\mathrm{C}$ banding locates the centromeres.

We have recently demonstrated that, at least as regards some chromosomes, there is little variation between individual human males in the localisation of chiasmata, ${ }^{12}$ and on this basis chiasma data have been used to construct genetic maps giving map 


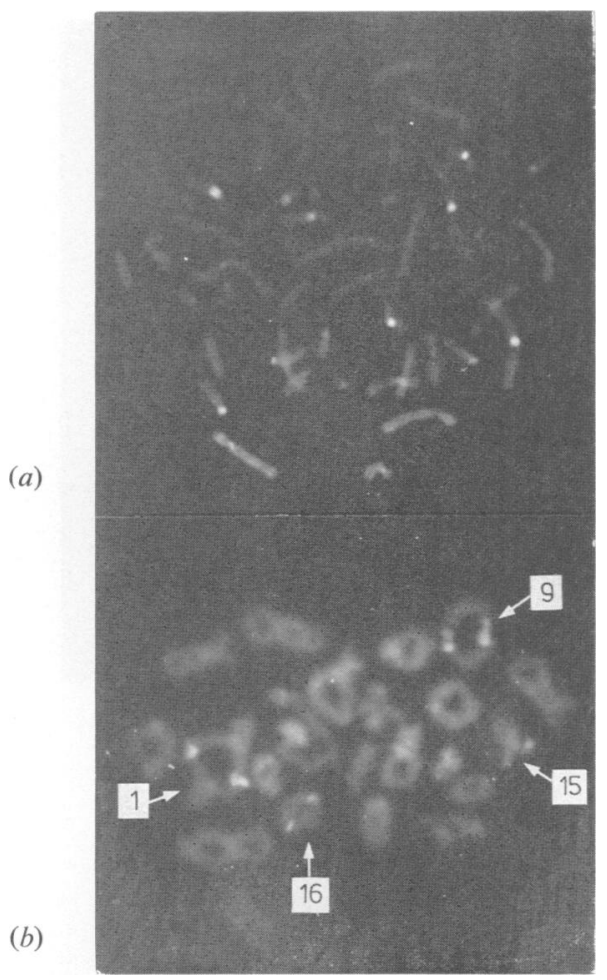

FIG 1 Distamycin A/DAPI stained cells. Note the bright fluorescence on chromosomes $1,9,15,16$, and the $Y$. (a) Mitotic cell (lymphocyte). (b) Meiotic cell at diakinesis.

distances and recombination fractions along the chromosome arms for chromosomes 1,2 , and $9 .{ }^{12}$ In this report, we present the chiasma distribution patterns on chromosomes 15 and 16 from two human males with normal mitotic 46,XY karyotypes, and compare these with those from one other normal male investigated previously. ${ }^{4}$ No significant interindividual variation was found and the chiasma data have been pooled to calculate male genetic map intervals and recombination fractions along the arms of these two chromosomes.

\section{Materials and methods}

Testicular biopsies were obtained during the investigation into the infertility of two patients, aged 27 and 29 years. Both had an apparently normal mitotic 46,XY karyotype and normal testicular histology. Slides were prepared by the air drying technique described by Hultén et $a l^{5}$ and then sequentially stained using quinacrine mustard, Distamycin A plus DAPI, cresyl fast violet, and finally $\mathrm{C}$ banding.
This order is essential and in our experience the sequence may not be altered.

Distamycin $\mathrm{A}$ is a non-fluorescent antibiotic which, when combined with the DAPI fluorochrome, reduces the overall fluorescence intensity of the chromosomes except for the heterochromatin of the secondary constrictions of chromosomes $1,9,16$, the short arm of chromosome 15, and the distal part of the long arm of the $Y$, which remain brightly fluorescent. ${ }^{6}$ There was no obvious difference between the DA-DAPI fluorescence of the diakinesis/ metaphase I chromosomes and that of the mitotic PHA stimulated lymphocytes (fig 1). Thus, chromosomes 1,9 , and 16 showed bright fluorescence of the secondary constrictions, corresponding to the constitutive heterochromatin, and in addition there was some fluorescence of the short arm of chromosome 15 and the distal part of the long arm of the $Y$. Neither of the two men investigated had any striking heteromorphism in their DA-DAPI fluorescence. Occasionally some other chromosomes showed fluorescent centromeres as is also seen at mitosis.

Cells analysed by the quadruple staining technique (fig 2) were projected at about 2500 times magnification, bivalents $1,9,15$, and 16 were drawn, and the centromere and chiasma positions measured using a digitiser. Chiasmata located within the most distal part of a chromosome arm, that is, corresponding to the width of the paired chromosomes, are referred to as 'terminal'. In some instances where the staining is faint, it is possible to see that such chiasmata are rarely terminal in the strict sense of the word. In other cases, however, heavy staining of the bivalents prevents elucidation of the precise location and we have therefore classified both these types of chiasmata as 'terminal'.

The chiasma patterns of chromosomes 1 and 9 were within the normal range of variation, indicating that the infertility of the two subjects investigated here is caused by factors other than those affecting chiasma formation. We have therefore considered them representative of the normal population as regards chromosomes 15 and 16 also.

The genetic distance between any two loci on a chromosome is normally expressed in centiMorgans (cM). A Morgan unit of genetic length is that length of a chromatid which has experienced, on average, one cross-over per meiotic division. Since each cross-over only affects two of the four chromatids, genetic map distances in Morgans were obtained as half the mean chiasma frequency between any two landmarks.

The corresponding recombination fractions are expressed in percentages. Each chiasma between two loci represents a single recombination event and is 

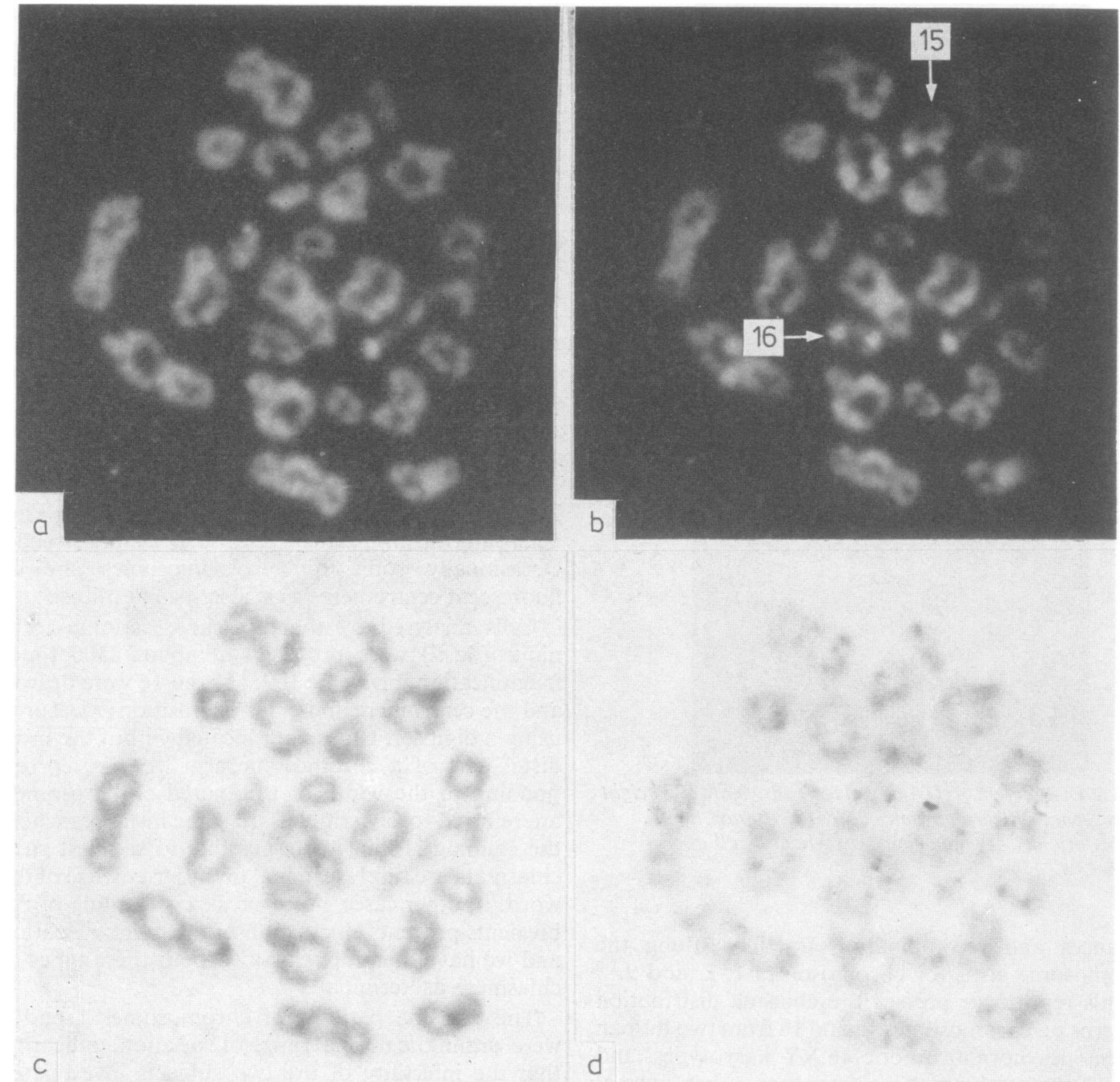

FIG 2 Meiotic cell at diakinesis stained consecutively by (a) quinacrine mustard to differentiate 16p from 16q;

(b) Distamycin A/DAPI to identify bivalents 15 and 16 easily; (c) cresyl fast violet to obtain a sharp outline of the chromosomes and chiasma position for drawing and measuring; and $(d) C$ banding for location of the centromere.

therefore expected to produce two recombinant and two non-recombinant gametes. Under the assumption that there is no chromatid interference, two or more chiasmata between the two loci will still produce $50 \%$ recombinant and $50 \%$ non-recombinant gametes. ${ }^{7}$ The recombination fractions were thus calculated as half the proportion of cells with at least one chiasma in the region concerned. The major bands of the mitotic chromosome as given in Francke and Oliver $^{8}$ were taken as landmarks, and the chiasma frequency of the corresponding parts of the meiotic chromosome were used for the calculations of genetic distance and recombination fraction. ${ }^{12}$

\section{Results}

MEAN CENTROMERE INDEX

The mean meiotic centromere index is $16 \cdot 2$ (SD 2.5) for bivalent 15 and 42.3 (SD 4.6) for bivalent 16, in good agreement with the results from mitotic cells and the measurements of Hultén. ${ }^{4}$

CHIASMA DATA

The numbers of bivalents analysed for chiasma frequency and chiasma distribution are given in table 1.

The mean chiasma frequency of the acrocentric 
chromosome 15 is 2.07 (SD 0.07). There were no chiasmata in the short arm $15 \mathrm{p}$. The sub-metacentric chromosome 16 has a mean chiasma frequency of 2.01 (SD 0.01 ) and the values for $16 \mathrm{p}$ and $16 \mathrm{q}$ are $1.01(\mathrm{SD} 0.01)$ and $1.00(\mathrm{SD} 0.00)$ respectively (table 2 ).

The distribution of chiasmata within chromosome

TABLE 1 Age of the individual males and number of cells analysed.

\begin{tabular}{|c|c|c|c|c|c|}
\hline \multirow[t]{2}{*}{ Male } & \multirow{2}{*}{$\begin{array}{l}\text { Cause of } \\
\text { investigation }\end{array}$} & \multirow[t]{2}{*}{$A g e^{\prime}$} & \multicolumn{2}{|c|}{ No of cells scored } & \multirow{2}{*}{$\begin{array}{l}S_{1} \cdot m b o l s \\
\text { in figs } \\
6 \text { and } 7\end{array}$} \\
\hline & & & $\begin{array}{l}\text { Chromo- } \\
\text { some } 15\end{array}$ & $\begin{array}{l}\text { Chromo- } \\
\text { some } 16\end{array}$ & \\
\hline HB--28 (case 1 ) & Infertility & 29 & 55 & 64 & $\star$ \\
\hline HB-36 (case 2) & Infertility & 27 & 23 & 28 & 0 \\
\hline H-173(Hultén $\left.{ }^{4}\right)$ & $\begin{array}{l}\text { Prostatic } \\
\text { cancer }\end{array}$ & 81 & 22 & 26 & A \\
\hline Total & & & 100 & 118 & \\
\hline
\end{tabular}

arms is given in the histograms in figs 3,4 , and 5 . No chiasmata were observed in the heterochromatic regions which were brightly fluorescent by the DADAPI technique, that is, there were no chiasmata in $15 p$ or $16 q h$. It is evident from the histograms that chiasmata are not uniformly distributed within chromosome arms, but are clustered within particular regions of the chromosome.

The chiasma pattern within bivalent $15 q$ is entirely different from that of bivalent 16 , in that proximal chiasmata occur much more often in the acrocentric chromosome 15 than in the sub-metacentric chromosome 16, but both show a strong tendency to form 'terminal' chiasmata (fig 3). The most common type of configuration in $15 \mathrm{q}$ is two chiasmata, one proximal and one distal (table 2). Occasionally there are three chiasmata, with the first counted from the centromere outwards being proximal, the second

TABLE 2 Chiasma frequencies for chromosomes 15 and 16. The proportion of each of the bivalent classes and the mean chiasma frequencies for these chromosomes are shown together with their mean centromere indices.

\begin{tabular}{|c|c|c|c|c|c|c|}
\hline \multirow[t]{2}{*}{ Bivalent } & \multirow{2}{*}{$\begin{array}{l}\text { Mean centromere } \\
\text { index }(S D)\end{array}$} & \multicolumn{2}{|l|}{ Bivalent classes } & \multicolumn{3}{|c|}{ Chiasma frequency $(S D)$} \\
\hline & & $\begin{array}{l}\text { No of chiasmata } \\
\text { per arm } \\
p q\end{array}$ & $\begin{array}{l}\text { Frequency of } \\
\text { cells }(\%)\end{array}$ & Total & $p$ & $q$ \\
\hline & & $0: 1$ & $2 \cdot 6$ & & & \\
\hline 15 & $16 \cdot 2(2 \cdot 5)$ & $0: 2$ & $\begin{array}{r}89 \cdot 7 \\
7.7\end{array}$ & $2.07(0.07)$ & - & $2.07(0.07)$ \\
\hline 16 & $42 \cdot 3(4 \cdot 6)$ & $\begin{array}{l}0: 3 \\
1: 1 \\
2: 1\end{array}$ & $\begin{array}{r}7 \cdot 7 \\
98 \cdot 9 \\
1 \cdot 1\end{array}$ & $2 \cdot 01(0 \cdot 01)$ & $1 \cdot 01(0.01)$ & $1.00(0.00)$ \\
\hline
\end{tabular}


HG 3 Chiasma distribution within chromosomes 15 and 16. The histograms represent the pooled chiasma data from the two males $H B-28$ and $H B-36$ and give the total number of chiasmata along the chromosome arms. The sections correspond to $10 \%$ of the length of the q arms. Note the proximal peak adjacent to the centromere in $15 q$ in comparison to the absence of chiasmata in the pericentromeric regions of chromosome 16. 

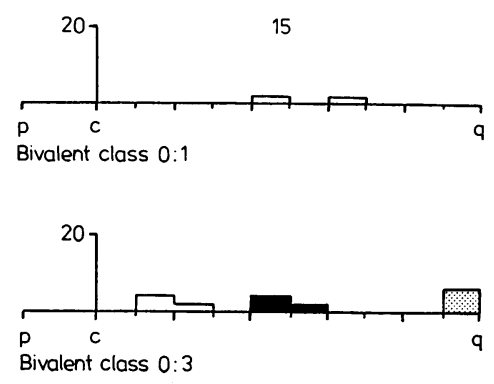

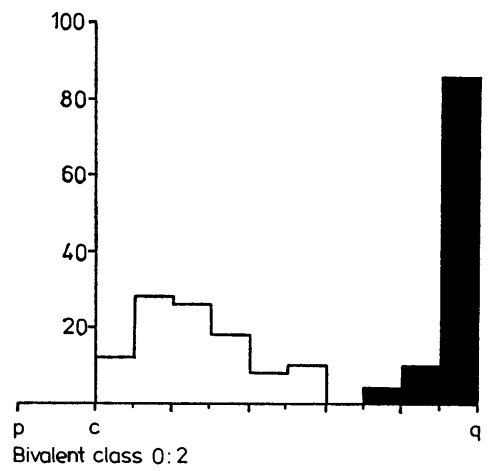

(a)
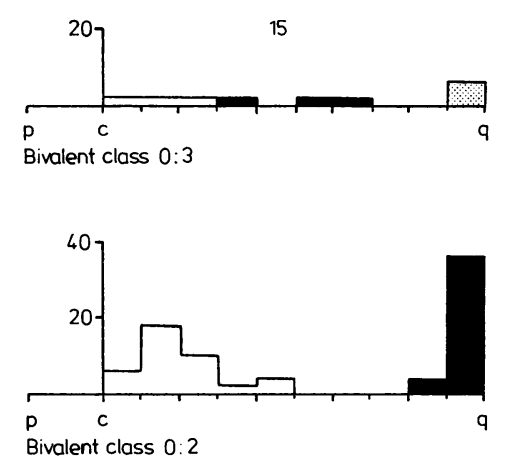

(b)

FIG 4 Chiasma distribution within chromosome 15 for the two males. (a) HB-28. Each bivalent class is given separately with single chiasma bivalents (bivalent class $0: 1)$ at the top, triple chiasma bivalents $(0: 3)$ in the middle, and double chiasma bivalents $(0: 2)$ at the bottom. (b) HB-36 with triple chiasma bivalents $(0: 3)$ at the top and double chiasma bivalents $(0: 2)$ at the bottom.

$\square$ First chiasma counted from the centromere outwards.

Second chiasma counted from the centromere outwards.

Third chiasma counted from the centromere outwards.

medial, and the third distal (almost always 'terminal'). Bivalents with only one chiasma are rarely seen, and in these the chiasma is usually medially located.

Chromosome 16 had one chiasma in each arm, which was most often 'terminally' located. One exceptional cell had two chiasmata in the short arm of chromosome 16, one proximal and one distal, in addition to the one in the long arm.

A statistical analysis using the KolmogorovSmirnov two sample test $^{910}$ demonstrated no significant difference in chiasma pattern between the two males as regards chromosomes 15 or 16 , and neither showed any difference from the pattern described for the single male analysed by Hultén. ${ }^{4}$ The $\mathrm{K}$ values range from $0 \cdot 15$ to $1 \cdot 26$, which are not significant at the $5 \%$ level. In the following calculations, therefore, the data from all three subjects have been pooled.

The total chiasma derived genetic length of chromosome $15 \mathrm{q}$ is $100.9 \mathrm{cM}$. Chromosome 16 is marginally longer, $102 \cdot 8 \mathrm{cM}$, with the short arm slightly longer genetically $(52.2 \mathrm{cM})$ than the long arm $(50.6 \mathrm{cM})$. To illustrate the general pattern of recombination, the genetic map distances between $ᄋ$ the centromere and points along the chromosome arms corresponding to $10 \%$ intervals of physical 을 length have been plotted in fig $6 a$ and $b$. In $15 q$ there is a rise up to $60 \%$ of the arm length, followed by a flattening in the 60 to $90 \%$ interval caused by a lower chiasma frequency, and then a sharp increase $\Omega$ towards the end of the arm owing to the high chiasma $N$ frequency in this region (fig 6a). In contrast, both $\underset{\mathrm{C}}{\mathcal{N}}$ arms of chromosome 16 show relatively much slower rises with steep increases only in the last $10 \%$ intervals (fig 6b).

The cumulative recombination fraction of $15 \mathrm{q} \stackrel{\mathscr{P}}{?}$ increases up to about $70 \%$ of the chromosome arm 7 by which point at least one chiasma has always $\stackrel{+}{\circ}$ occurred, and accordingly the recombination $\stackrel{\mathbb{D}}{\circ}$ fraction is $50 \%$ (fig 7a). It should be noted, however, $\stackrel{\vec{\Phi}}{\stackrel{2}{2}}$ that the cumulative recombination fraction and $\frac{0}{\sigma}$ 
(a)

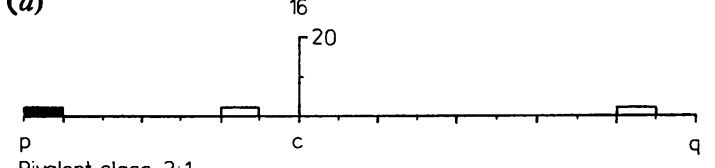

Bivalent class 2:1

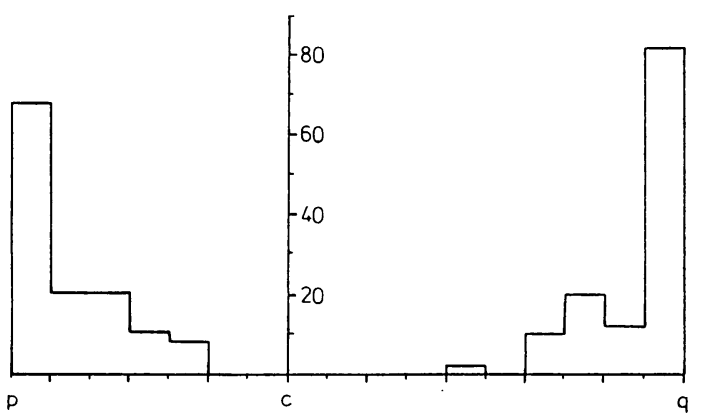

Bivalent class 1.1

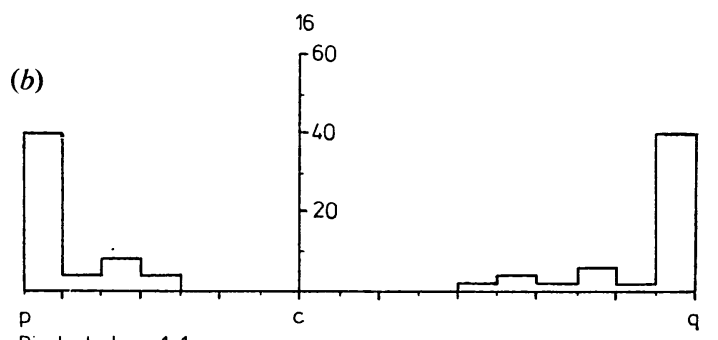

Bivalent class 1:1
FIG 5 Chiasma distribution within chromosome 16 for the two males. (a) HB-28 with triple chiasma bivalent (bivalent class 2:1) at the top and double chiasma bivalents (1:1) at the bottom. (b) HB-36 with double chiasma bivalents (1:1) only. Note similarities between the two subjects in chiasma pattern in double chiasma bivalents.

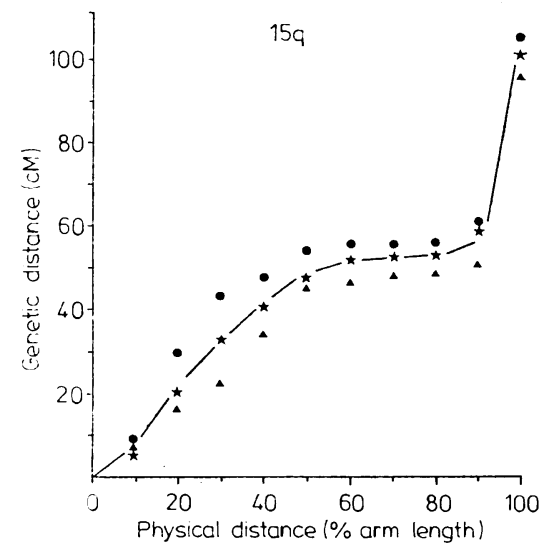

(a)

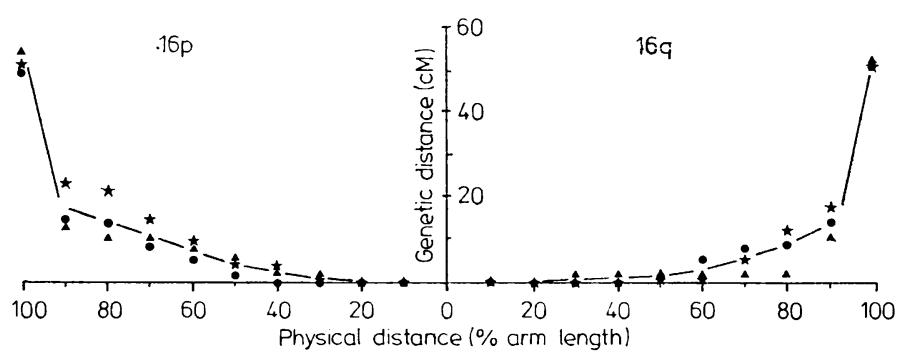

(b)

FIG 6 Chiasma derived cumulative genetic distances between the centromere and $10 \%$ intervals along chromosome $15 q(a)$ and chromosome $16 p$ and $16 q(b)$. The values for each of the three men are given separately, the line representing the mean. $\star$ Case 1 . $\bullet$ Case 2 . \Hultén. ${ }^{4}$ 


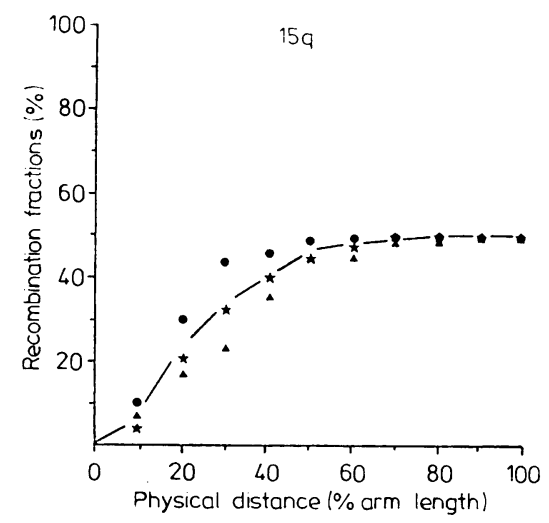

(a)

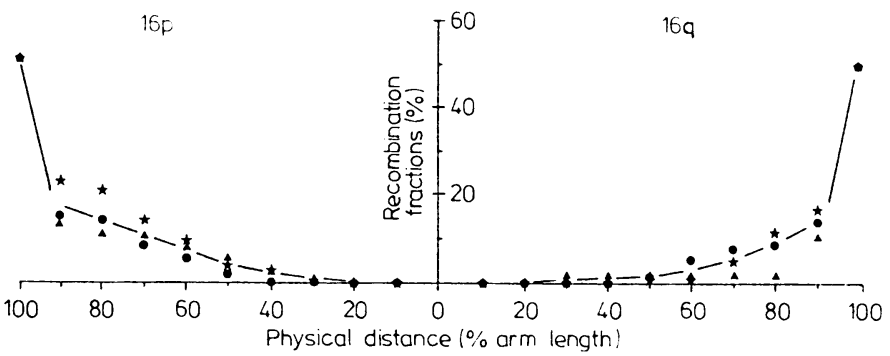

(b)

FIG 7 Chiasma derived recombination fractions between the centromere and 10\% intervals along chromosome $15 q(a)$ and chromosome $16 p$ and $16 q(b)$.

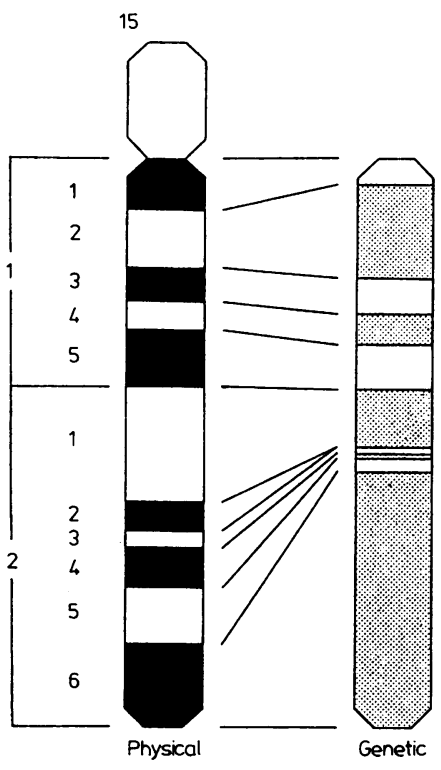

(a)
16

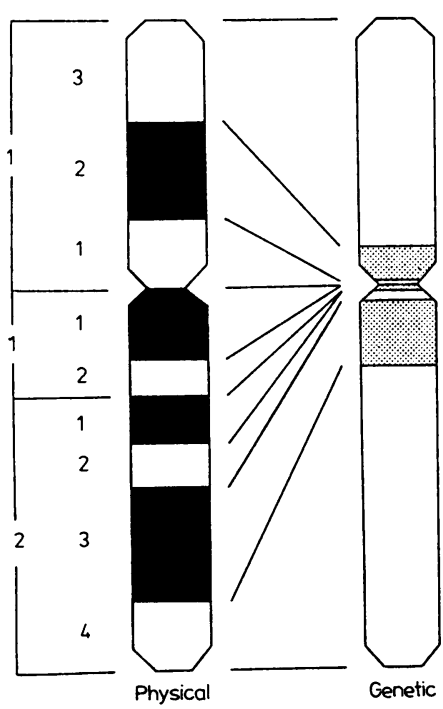

(b)
FIG 8 Relationship between physical and genetic length of $(a)$ chromosome 15 and (b) chromosome 16. The physical map gives the major bands as measured by Francke and Oliver. ${ }^{8}$ The genetic map is derived from the chiasma frequency of the corresponding parts of the meiotic chromosomes. genetic lengths have the same numerical values only up to about $40 \%$ of the $15 \mathrm{q}$ arm length, and that beyond this point recombination fractions show lower values. By comparison, the recombination fractions of $16 \mathrm{p}$ and $16 \mathrm{q}$ increase more slowly and do not reach $50 \%$ until the end of the chromosome arm (fig $7 b$ ). The recombination values in $16 \mathrm{q}$ do not diverge from those of genetic distance, since this arm has invariably one chiasma.

The relationship between physical and genetic lengths is further illustrated in fig 7 , giving the genetic map intervals of chromosomes 15 (fig 8a) and 16 (fig $8 \mathrm{~b}$ ) corresponding to the major band borders of
Francke and Oliver. ${ }^{8}$ The high numbers of chiasmata occurring distally and 'terminally' cause a $\sigma$ dramatic genetic extension of the bands 15q26, N $16 \mathrm{p} 13$, and $16 \mathrm{q} 24$, while the remaining parts of N chromosome $16(16 \mathrm{p} 11 \rightarrow 12$ plus $16 \mathrm{q} 11 \rightarrow 23)$ show very little recombination. In contrast, the proximal part of $15 \mathrm{q}$ (bands $15 \mathrm{q} 12 \rightarrow 14$ ) has a higher level of $\stackrel{\varrho}{\mathcal{D}}$ recombination than the medial part (bands 15q15 $\rightarrow$ @ 25).

The genetic distances between band borders and their corresponding recombination fractions estimated from the chiasma data are given in table $3 . \stackrel{\mathbb{D}}{\mathbb{D}}$ The numbers are presented in such a way that the 


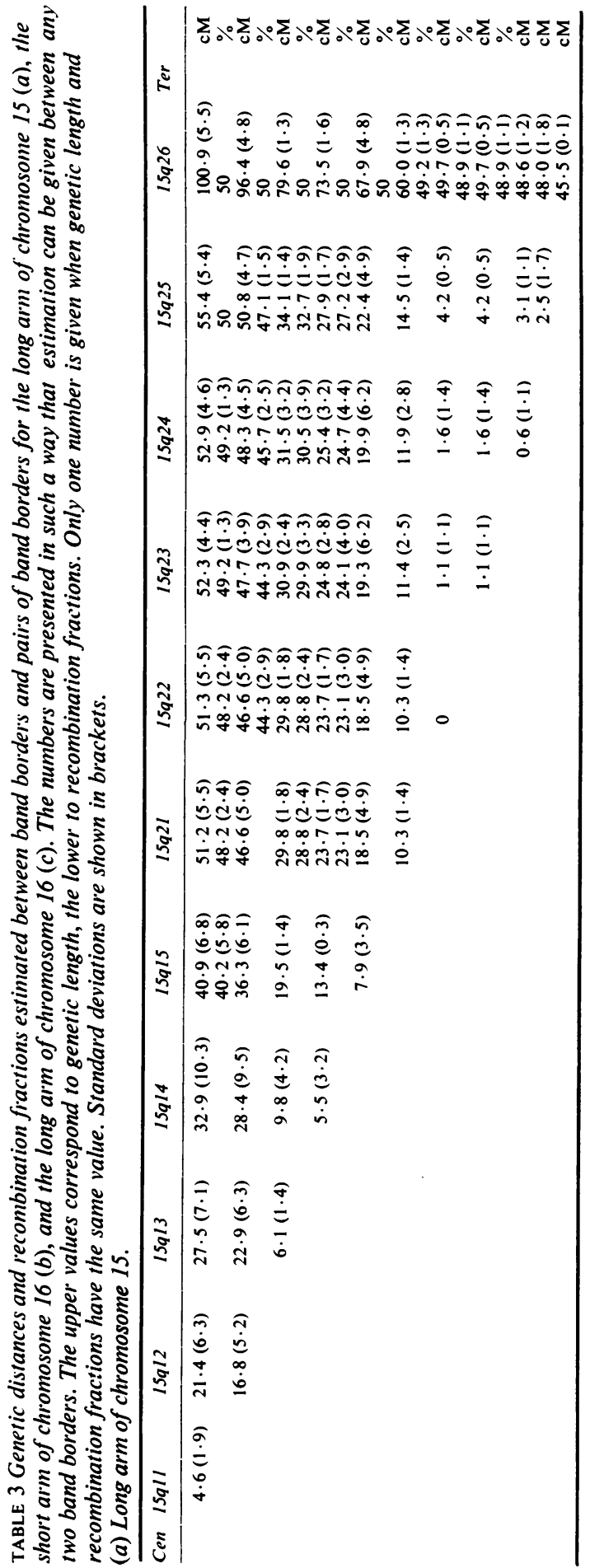

(b) Short arm of chromosome 16.

\begin{tabular}{lllll}
\hline Cen & $16 p 11$ & $16 p 12$ & $16 p 13$ & Ter \\
\hline & $0.9(0 \cdot 9)$ & $7.4(2 \cdot 0)$ & $52 \cdot 2(3 \cdot 1)$ & $\mathrm{cM}$ \\
& & 50 & $\%$ \\
& $6 \cdot 6(1 \cdot 7)$ & $51 \cdot 3(2 \cdot 2)$ & $\mathrm{cM}$ \\
& & 50 & $\%$ \\
& & $44 \cdot 7(3 \cdot 3)$ & $\mathrm{cM}$ \\
& & $44 \cdot 1(2 \cdot 4)$ & $\%$ \\
\hline
\end{tabular}

values between any two band borders may be obtained. For example, in table 3a, the genetic length of band $15 \mathrm{q} 11$ is $4.6 \mathrm{cM}$ (SD 1.9) and that of band $15 \mathrm{q} 21$ is $10.3 \mathrm{cM}$ (SD 1.4). The cumulative genetic distance between the centromere and the distal border of band $15 \mathrm{q} 21$ is $51.2 \mathrm{cM}$ (SD 5.5) and the corresponding recombination fraction is $48.2 \%$ (SD 2.4). Similarly, the genetic distance between the proximal border of band $15 \mathrm{q} 21$ and the distal border of band $15 \mathrm{q} 23$ is $11.4 \mathrm{cM}$ (SD 2.5), but in this instance the recombination fraction has the same numerical value.

\section{Discussion}

We have chosen to compare the chiasma distribution patterns of bivalents 15 and 16 not only because these chromosomes happen to be easily identified by the DA-DAPI staining technique but also because this comparison serves to illustrate the influence of arm ratio on the chiasma locations and thus on patterns of recombination. Chromosomes 15 and 16 are of roughly equal physical length and have almost the same mean number of chiasmata (table 1). Yet the distribution along the chromosome arms is entirely different, the nearly metacentric chromosome 16 almost always having two chiasmata, one in each arm, located distally/ 'terminally', while the acrocentric chromosome 15 shows more variation with either one, two, or three localised chiasmata in the long arm and none in the short arm (fig 4).

In the situation where the long arm of chromosome 15 has three chiasmata, one is always located adjacent to the centromere. Therefore, the idea commonly expressed that chiasmata in the human never occur in the vicinity of the centromere should be refuted. In the human male, this is generally true for non-acrocentric chromosomes and the short acrocentrics (in the majority of cells), but it is not true for the long acrocentrics, where there is, in fact, a prominent proximal peak near the centromere. Our interpretation of this situation is that these chiasmata are preferentially formed in this particular part of the chromosome, that is, they are 'localised'.11 
(c) Long arm of chromosome 16.

\begin{tabular}{|c|c|c|c|c|c|c|c|c|}
\hline Cen & $16 q 11$ & $16 q 12$ & $16 q 21$ & $16 q 22$ & $16 q 23$ & $16 q 24$ & Ter & \\
\hline & \multirow[t]{3}{*}{0} & $0.6(1 \cdot 1)$ & $0.6(1.1)$ & $2 \cdot 1(1 \cdot 4)$ & $10 \cdot 5(5 \cdot 7)$ & \multirow{3}{*}{$\begin{array}{l}50 \cdot 6(1 \cdot 1) \\
50 \\
50 \cdot 6(1 \cdot 1) \\
50 \\
50 \\
50 \\
48 \cdot 6(1 \cdot 9) \\
40 \cdot 2(6 \cdot 8)\end{array}$} & & \multirow{3}{*}{$\begin{array}{l}\mathrm{cM} \\
\% \\
\mathrm{cM} \\
\% \\
\mathrm{cM} \\
\mathrm{cM} \\
\mathrm{cM} \\
\mathrm{cM}\end{array}$} \\
\hline & & $0 \cdot 6(1 \cdot 1)$ & $0 \cdot 6(1 \cdot 1)$ & $2 \cdot 1(1 \cdot 4)$ & $10 \cdot 5(5 \cdot 7)$ & & & \\
\hline & & & 0 & $\begin{array}{l}1.45(1.9) \\
1.45(1.9)\end{array}$ & $\begin{array}{l}9.8(6 \cdot 8) \\
9.8(6 \cdot 8) \\
8.4(5 \cdot 6)\end{array}$ & & & \\
\hline
\end{tabular}

Other examples of chiasmata interpreted as 'localised' are the distal peaks in 15q, 16p, and 16q (fig 3). As discussed in more detail in the previous articles in this series, ${ }^{12}$ we find it unlikely that the clustering of chiasmata is a result of movement from their original positions, particularly in view of the recent BrdU labelling experiments ${ }^{12} 13$ which, in a variety of organisms, demonstrate a correspondence between label exchange and chiasma position.

Having described the general features of the chiasma distribution, one wonders about variation between persons. A certain amount of interindividual variation might be expected when dealing with a biological parameter which must be under genetic control. From this viewpoint, it is remarkable that the two men investigated here show almost identical patterns of chiasma distribution in bivalents 15 and 16 and that this pattern is indistinguishable from that of the only male investigated previously. ${ }^{4}$ The lack of inter-individual variation in chiasma distribution on bivalents 15 and 16 is also reflected in the homogeneity of cumulative genetic length along the chromosome arms (fig 6a, b), and the same holds true for the recombination fraction between the centromere and points along the chromosome arm (fig 7a, b).

The explanation for the extended genetic length in the distal part of $15 \mathrm{q}$, although full recombination is already reached at $70 \%$ of the way along the arm, is that the genetic map distance is estimated as half the mean number of chiasmata, while the recombination fraction is calculated as the proportion of cells having at least one chiasma in the region concerned. Under the assumption that there is no chromatid interference, the recombination fraction then remains unchanged with the occurrence of a second or third chiasma. On the other hand, no striking discrepancies are observed between these parameters in chromosome 16 because here there is nearly always a single chiasma in each arm.

The genetic maps in fig 8 serve further to illustrate the non-randomness of the distribution of recombination along the chromosome arms. A glance at these maps suggests that it may be misleading to equate genetic lengths directly with the number of genes $^{14}$ or with the DNA content. ${ }^{15}$ If our assumptions that chiasmata at diakinesis/metaphase $\mathbf{I}_{0}$ directly reflect crossing-over sites are true, then, for example, the proximal $50 \%$ of $16 \mathrm{q}$, which contains approximately $25 \mathrm{Mbp}$ of DNA, has a genetic length of only $2 \cdot 1 \mathrm{cM}$ (table $3 \mathrm{c}$ ), while the value for the 8

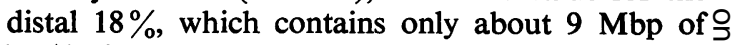
DNA, is $40.2 \mathrm{cM}$. Here a section of chromosomewhich is one third of the length of another is expected to have 20 times as much recombination.

It should be borne in mind, however, that this $\stackrel{\text { ? }}{+}$ particular example deals with the situation in the $\overrightarrow{0}$ human male and we expect that the chiasma distribution, and therefore the recombination pattern, is quite different in the female.

A grant received from the Lebanese University, Faculty of Science, is gratefully acknowledged. We $\stackrel{\varnothing}{\varnothing}$ would like to thank Dr Gareth Jones, Genetics $\varrho$ Department, University of Birmingham for his $\overrightarrow{\overrightarrow{0}}$ helpful comments on the manuscript and David Laurie and Rodger Palmer from this laboratory for valuable discussion.

\section{References}

1 Hultén MA, Palmer RW, Laurie DA. Chiasma derived genetic maps and recombination fractions: chromosome $\delta$ 1. Ann Hum Genet 1982;46:167-75.

2 Laurie DA, Palmer RW, Hultén MA. Chiasma derived genetic lengths and recombination fractions: chromo- $\frac{\text { O }}{3}$

somes 2 and 9. Ann Hum Genet 1982;46:233-44.
8 Caspersson T, Hultén M, Lindsten J, Zech L. Identification of chromosome bivalents in human male meiosis by quinacrine mustard fluorescence analysis. Hereditas $\mathrm{N}$ $1971 ; 67: 147-9$.

4 Hultén M. Chiasma distribution at diakinesis in the $\mathrm{N}$ normal human male. Hereditas $1974 ; 76: 55-78$.

5 Hultén M, Lindsten J. Cytogenetic aspects of human $\omega$ male meiosis. Adv Hum Genet 1973;4:327-87.

- Schweizer D, Ambros P, Andrle M. Modification of 0 DAPI banding on human chromosomes by prestaining with a DNA-binding oligopeptide antibiotic, Distamycin A. Exp Cell Res $1978 ; 3: 327-32$.

7 Mather K. Crossing-over. Biol Rev 1938;13:252-92. T

8 Francke W, Oliver N. Quantitative analysis of high resolution trypsin-Giemsa bands on human prometaphase chromosomes. Hum Genet 1978;45:137-65.

9 Meddis R. Statistical handbook for non-statisticians. London: McGraw-Hill, 1975. 
10 Shaw DD, Knowles GR. Comparative chiasma analysis using a computerized optical digitizer. Chromosoma 1976; 59:103-27.

11 Rees H, Jones RN. Chromosome genetics. London: Arnold, 1977.

12 Tease C, Jones GH. Analysis of exchanges in differentially stained meiotic chromosomes of Locusta migratoria after BrdU-substitution and FPG staining. I. Crossover exchanges in monochiasmate bivalents. Chromosoma 1978;69:163-78.

13 Kanda N, Kato H. Analysis of crossing-over in mouse meiotic cells by BrdU labelling technique. Chromosoma $1980 ; 78: 113-21$.
14 McKusick VA. The human gene map newsletter. 1 December 1981 .

15 Ruddle FH. A new era in mammalian gene mapping: somatic cell genetics and recombinant DNA methodologies. Nature $1981 ; 294: 115-20$.

Correspondence and requests for reprints to $\mathrm{Dr} N$ Saadallah, Regional Cytogenetics Laboratory, East Birmingham Hospital, Bordesley Green East, Birmingham B9 5ST. 